\title{
CORRESPONDENCE
}

\section{Early discharge and outpatient treatment of patients admitted for acute pulmonary embolism}

\section{To the Editor:}

We read with great interest the study by MoORES et al. [1]. The authors propose that patients admitted for acute pulmonary embolism and classified as Pulmonary Embolism Severity Index (PESI) class III could be more appropriately risk-stratified by recalculating the PESI score $48 \mathrm{~h}$ after admission (PESI48). 83 out of 304 PESI class III patients were reclassified as low risk (defined as PESI48 class I and II) while 16 were reclassified into classes IV or V, which, according to the authors, led to an overall net improvement in risk reclassification estimated at $54 \%$. Patients re-categorised as low risk had a mortality rate of $1.2 \%$ as opposed to $11.3 \%$ in those who remained high risk (PESI $48 \geqslant \mathrm{III}$ ).

Prognostic assessment of patients with acute pulmonary embolism is of pivotal importance and still an area of ongoing research. Good prognostication of pulmonary embolism is extremely costeffective, as demonstrated by AUJESKY et al. [2], who found low molecular weight heparin treatment of pulmonary embolism to be cost-saving if $\geqslant 8 \%$ of patients were eligible for early discharge or if $\geqslant 5 \%$ of patients could be treated as outpatients.

Although renewed statistical approaches and more efficient scores in low- and high-risk patient stratification are welcome, some authors have already given an outstanding contribution in this matter. A score developed for the selection of the lowest-risk patients eligible for outpatient treatment should aim to have high sensitivity and negative predictive value. ERKENS et al. [3] suggested the PESI and Simplified PESI (sPESI) scores could accurately identify patients with acute pulmonary embolism who were at low and high risk for short-term adverse events, while JIMÉNEZ et al. [4] concluded PESI had higher discriminative performance than the Geneva score and allowed a more appropriate selection of patients with very low adverse event rates during the initial days of acute pulmonary embolism therapy. Moores et al. [5], who had already focused on this subject, reported troponin I values did not add prognostic power to PESI in terms of low-risk patient selection. To our knowledge, the Low-Risk Pulmonary Embolism Decision (LR-PED) score is the only risk model derived from a sample of haemodynamically stable patients without any sign or evidence of extensive myocardial necrosis, or clinical or echocardiographic right ventricular dysfunction (those patients potentially suitable for early discharge and outpatient treatment) [6]. However, clinical implementation of the LR-PED model is dependent on its potential validation in larger cohorts of low-risk pulmonary embolism patients.

MoORES et al. [1] add new data to their already renowned contribution in this area of research. Some of the results of their study had never been reported before and, therefore, must be highlighted, as follows. 1) Calculation of PESI48 or sPESI48 has significant additional prognostic value. 2) PESI48 and sPESI48 have apparently higher sensitivity and negative predictive value than PESI and SPESI, respectively. 3) Patients categorised as low risk by PESI48 and especially sPESI 48 are truly low-risk patients; in this regard, sPESI48 seems more attractive in its ability to truly identify patients eligible for early discharge and outpatient treatment. 4) Patients re-classified by PESI48 as classes IV-V are at very high mortality risk and might benefit from additional therapeutic measures.

Some additional considerations should be stated, however, as follows. 1) It would be interesting if the authors could assess more thoroughly the performance of their PESI48 and sPESI48 models. Reporting discrimination through receiver operating characteristic (ROC) curves, calibration using the Hosmer-Lemeshow goodness-of-fit test and accuracy within each individual patient with the Brier score could add robustness to their theses in case of confirmation of the high performance of PESI48 and sPESI48. 2) Stating a $54 \%$ net improvement in risk reclassification may give the false notion that the PESI48 model is substantially better than PESI when, in reality, these two models were not being directly compared (as all patients in the sample were PESI class III). A straight and honest comparison between both models would be worthwhile (PESI at admission versus PESI48). The authors demonstrated that PESI48 correctly reclassified a significant proportion of PESI class III patients in more appropriate risk categories, but we do not know whether PESI48 would lead to a significant net improvement in risk reclassification of patients other than PESI class III (this was not addressed in their study). 3) PENCINA et al. [7] suggest integrated differences in sensitivities and "one minus specificities", and their difference, as other measures of improvement in performance offered by the new marker or risk model (in this case, PESI48; the so-called integrated discrimination improvement). The authors report negative integrated discrimination improvement (IDI) values, which contradict their positive net reclassification improvement (NRI). For very large or very small differences in performance, improvement in the area under a ROC curve, IDI and NRI should yield the same conclusions [7].

In summary, the 48-h re-calculation of PESI or sPESI scores in patients admitted for acute pulmonary embolism may help identify truly low-risk patients who could be eligible for early discharge and outpatient treatment. Potential implementation of this strategy should be preceded by its prospective validation and a more rigorous model performance evaluation through means of discrimination, calibration and re-classification measures.

Sérgio Nuno Craveiro Barra, Luís Paiva and Rui Providência Cardiology Dept, Coimbra Hospital and University Centre, Hospital Geral, Coimbra, Portugal. 
Correspondence: S.N.C. Barra, Cardiology Dept, Coimbra Hospital and University Centre, Hospital Geral, Quinta dos Vales, 3041-801 S. Martinho do Bispo, Coimbra, Portugal. E-mail: sergioncbarra@gmail.com

Statement of Interest: None declared.

\section{REFERENCES}

1 Moores L, Zamarro C, Gómez V, et al. Changes in PESI score predict mortality in intermediate-risk patients with acute pulmonary embolism. Eur Respir J 2013; 41: 354-359.

2 Aujesky D, Smith KJ, Cornuz J, et al. Cost-effectiveness of lowmolecular-weight heparin for treatment of pulmonary embolism. Chest 2005; 128: 1601-1610.

3 Erkens PM, Gandara E, Wells PS, et al. Does the Pulmonary Embolism Severity Index accurately identify low risk patients eligible for outpatient treatment? Thromb Res 2012; 129: 710-714.

4 Jiménez D, Yusen RD, Otero R, et al. Prognostic models for selecting patients with acute pulmonary embolism for initial outpatient therapy. Chest 2007; 132: 24-30.

5 Moores L, Aujesky D, Jiménez D, et al. Pulmonary Embolism Severity Index and troponin testing for the selection of low-risk patients with acute symptomatic pulmonary embolism. J Thromb Haemost 2010; 8: 517-522.

6 Barra S, Paiva L, Providência R, et al. LR-PED rule: Low Risk Pulmonary Embolism Decision Rule - a new decision score for low risk pulmonary embolism. Thromb Res 2012; 130: 327-333.

7 Pencina MJ, D'Agostino RB Sr, D'Agostino RB Jr, et al. Evaluating the added predictive ability of a new marker: from area under the ROC curve to reclassification and beyond. Stat Med 2008; 27: 157-172.

DOI: $10.1183 / 09031936.00108112$

\section{From the authors:}

We would like to thank S.N.C. Barra and co-workers for their thoughtful response to our article [1]. We agree with the authors that prognostic assessment of patients with acute pulmonary embolism is of pivotal importance and, thus, is an area of active investigation for our group. We would like to comment on just a few of the points raised by the authors. First, the authors comment that the Low-Risk Pulmonary Embolism Decision score is the only model derived completely from haemodynamically stable patients, which they define as those without any evidence of myocardial necrosis or echocardiographic right ventricular dysfunction (RVD). We agree that risk stratification tools should be derived from haemodynamically stable pulmonary embolism patients. However, we disagree that markers of myocardial injury and RVD should be performed prior to clinical scores. In fact, there is increasing evidence that the Pulmonary Embolism Severity Index (PESI) and Simplified PESI (sPESI) identify low-risk patients who might benefit from outpatient therapy of their disease without the need for echocardiography or troponin tests $[2,3]$.

S.N.C. Barra and co-workers seem to take away from our study that the PESI score $48 \mathrm{~h}$ after admission (PESI48) and sPESI48 are more accurate than the PESI and sPESI. This is not really true. As the authors note later in their correspondence, we did not compare PESI with PESI48 but retested PESI in those who were initially PESI class III. We were not interested in finding a score better than PESI but rather a way to increase our ability to identify patients who will have a good outcome regardless of the treatment setting. Thus, our intention in the current study was to further risk-stratify patients who are at intermediate risk on presentation. In essence, calculation of PESI48 can identify a portion of intermediate risk patients who have responded favourably to their initial treatment and can be safely discharged early from the hospital. From a clinical application standpoint, we would suggest that PESI and/or sPESI can be used to identify a very low-risk population that can be treated entirely as outpatients. Calculation of the PESI48/sPESI48 can then be used to identify patients appropriate for early discharge after an initial hospitalisation.

The authors asked us to consider further assessment of PESI48 by calculating discrimination, calibration and accuracy scores. We have gladly done so. For PESI48, the area under the curve is 0.75 (95\% CI 0.66-0.84), Hosmer-Lemeshow goodness-of-fit is $5.38(p=0.72)$ and Brier score is 0.073 . Finally, we thank the authors for identifying a typographical error in our manuscript. They note that we reported a negative integrative discrimination index (IDI). In fact, the IDI was positive. In other words, the new score improves classification by $2 \%$. This error has been corrected in this issue of the European Respiratory Journal, both in print and online.

Thank you again for the correspondence and the opportunity to further clarify our findings.

\section{Lisa K. Moores*, Celia Zamarro*, Vicente Gómez ", Drahomir Aujesky ${ }^{+}$, Leticia García\#, Rosa Nieto", Roger Yusen ${ }^{\S}$ and David Jiménez ${ }^{\#}$ on behalf of the Instituto Ramón y Cajal de Investigación Sanitaria Pulmonary Embolism Study Group}

*F. Edward Hebert School of Medicine, Uniformed Services University of the Health Sciences, Bethesda, MD, and ${ }^{\S}$ Divisions of Pulmonary and Critical Care Medicine and General Medical Sciences, Washington University School of Medicine, St Louis, MO, USA. "Respiratory Dept, Ramón y Cajal Hospital, and ${ }^{\top}$ Medicine Dept, Ramón y Cajal Hospital, Madrid, Spain. ${ }^{+}$Division of General Internal Medicine, Bern University Hospital, Bern, Switzerland.

Correspondence: L.K. Moores, Uniformed Services University of the Health Sciences, Bethesda, MD 20814, USA. E-mail: lisa.moores@usuhs.edu

Statement of Interest: A statement of interest for R. Yusen is available at www.erj.ersjournals.com/site/misc/statements. xhtml

\section{REFERENCES}

1 Moores L, Zamarro C, Gómez V, et al. Changes in PESI score predict mortality in intermediate-risk patients with acute pulmonary embolism. Eur Respir J 2013; 41: 354-359.

2 Lankeit M, Jiménez D, Kostrubiec M, et al. Predictive value of the high-sensitivity troponin $\mathrm{T}$ assay and the Simplified Pulmonary Embolism Severity Index in hemodynamically stable patients with 